\title{
Squaring the circle? On the search for circulating biomarkers in polygenic psychiatric disorders
}

\author{
Johannes Hebebrand • Jochen Antel
}

Published online: 4 March 2014

(c) Springer-Verlag Berlin Heidelberg 2014

The DSM-5 criteria for ADHD have to a great extent retained the wording for the 18 clinical symptoms which were specified in DSM IV (APA 1994, 2013) indicating that our concept of this disorder has remained rather stable over the past decades [5]. For ADHD but also for other psychiatric disorders, diagnoses are based on a consensus about clusters of clinical symptoms. This categorization has focused on reliability, entailing the benefit that we have fewer problems agreeing on the label for an individual patient. However, this diagnostic procedure has been criticized for not allowing valid diagnoses [10]. As pointed out by the current NIMH director Thomas Insel, symptombased diagnoses have been replaced in other medical fields because they by themselves only rarely allow us to deduce the best choice of treatment. Blood tests represent a mainstay of the assessment of patients with somatic disorders; a mere blood sampling is required to allow numerous determinations of serum markers which are frequently pivotal for coming up with the correct diagnosis and treatment.

Based on this criticism of psychopathology based categorization of disorders, NIMH has launched the Research Domain Criteria (RDoC) project to transform diagnosis by incorporating genetics, imaging, cognitive science, and other levels of information to lay the foundation for a new classification system. A set of assumptions underlies this development: (1) the current DSM-5 criteria must not in any way constrain a diagnostic approach based on biology as well as symptomatology, (2) mental disorders are

J. Hebebrand $(\bowtie) \cdot J$. Antel

Department of Child and Adolescent Psychiatry, Psychosomatics

and Psychotherapy, Universitätsklinikum Essen, University of

Duisburg-Essen, Essen, Germany

e-mail: johannes.hebebrand@uni-due.de biologically based and involve brain circuits that implicate specific domains of cognition, emotion, or behavior, (3) each level of analysis needs to be understood across a dimension of function, and (4) mapping the cognitive, circuit, and genetic aspects of mental disorders will yield new and better targets for treatment [10].

Due to the diagnostic problems and uncertainties associated with ADHD, a more valid diagnosis of this most common childhood disorder would certainly be welcomed [19]. Biomarkers offer the opportunity to improve the diagnostic assessment and at the same time to provide a potential insight into etiological mechanisms. A biomarker has been defined as a characteristic that can be objectively measured and evaluated as an indicator of a normal biological process, a pathogenic process, or a response to a therapeutic intervention [16]. Whereas potential ADHD biomarkers are by no means limited to circulating markers (see e.g. [12] for event-related potentials, respectively), serum (or plasma) biomarkers offer a readily viable opportunity to increase the validity of diagnostic decisions in psychiatry. However, the search for biomarkers of psychiatric disorders has largely proven elusive. Based on the stringent criteria (a) high specificity and sensitivity ( $>80 \%$ for both), (b) reliability, reproducibility, inexpensiveness, and easy use, and (c) independent confirmations by qualified researchers published in peer-reviewed journals, a consensus report of the task force on biological markers of the World Federation of Societies of Biological Psychiatry (WFSBP) and the World Federation of ADHD concluded that no ADHD biomarker can be identified [18]. Clinical usefulness certainly depends on high specificity and sensitivity; thus, the detection of serum antibodies against glutamic acid decarboxylase 65 in roughly $25 \%$ of children with ADHD [13], but also in patients with autism or neurological disorders precludes the notion that such 
antibodies are in any way specific for ADHD. Research into the identification of novel biomarkers for ADHD is ongoing via the application of the associated technologies for the identification of protein biomarker signatures in neurodevelopmental disorders [6].

Scassellatti et al. [14] conducted a systematic literature search for the time period 1968-2011 for peripheral ADHD biomarkers and performed meta-analyses of the thus identified 210 case-control studies. 71 studies pertained to the main metabolites and metabolism enzymes of the monoaminergic neurotransmission pathway, 87 studies to environmental risk factors divided into heavy metals (18 studies), substance/chemical exposures (16 studies), and nutritional factors (trace elements: 29 studies; essential fatty acids: 24 studies), and 22 studies to the hypothalamic-pituitary-adrenal axis (HPA) pathway; 31 studies did not fit a category. The meta-analyses based on the availability of drug-naïve/free case-control studies revealed five statistically significant comparisons for norepinephrine, 3-methoxy-4-hydroxyphenylethylene glycol, monoamine oxidase, zinc, and cortisol, respectively. The effect sizes for three of these biomarkers differed substantially between studies. Suggestive findings were obtained for neuropeptide Y, manganese and dehydroepiandrosterone. The authors deemed further studies necessary to assess specificity and clinical usefulness.

In this issue of European Child and Adolescent Psychiatry, Scassellati et al. [15] assessed serum levels of brain derived neurotrophic factor (BDNF) in 45 drugnaïve ADHD children and 45 healthy subjects matched for age and sex. The investigators carefully delineate the rationale behind their choice of the neurotrophin BDNF as a potential biomarker including data from rodent, biochemical, imaging, genetic and pharmacological studies. A previous study had found elevated levels of plasma BDNF in children with ADHD [17]. In contrast, in an adult study encompassing 54 cases and 59 controls, BDNF serum levels were found to be substantially lower in ADHD [3].

In their current study, Scassellati et al. diagnosed ADHD according to DSM IV, exclusion criteria for the controls are described. All subjects seemingly had a similar ethnic background. Blood sampling was performed after an overnight fast between 8 and 9 a.m.; the procedure for determination of serum BDNF levels appears sound. Patients and controls did not differ significantly with respect to their serum levels; the difference in mean levels was merely $0.5 \mathrm{ng} / \mathrm{ml}$. Interestingly, the standard deviation was substantially larger in controls as compared to cases $(38.82 \pm 8.29$ vs. $39.3 \pm 0.41 \mathrm{ng} / \mathrm{ml})$. No differences were observed according to ADHD type. The authors discuss their negative results in light of similarly negative data for BDNF single nucleotide polymorphisms (SNPs) derived from meta-analysis of genome-wide association studies (GWAS) for ADHD [20].

Can we deduce information on serum markers for psychiatric disorders from such genetic studies? If a locus reaches genome-wide significance, it would certainly be of interest to link the finding to quantitative expression data of the gene underlying the association signal and if possible to determine serum levels of the respective protein. We hereby make the assumption that the peripheral level of a protein is somehow related to its function in the CNS. Because odds ratios for genome-wide significant SNPs only infrequently exceed 1.3 , we need to acknowledge that very large sample sizes might be required to pick up a significant difference of the level of such a protein between cases and controls. This applies even more so, if the SNP effects the transcription rate by just a few percent-a scenario, which by no means is unlikely. In this context, current biomarker studies clearly appear underpowered. However, if the differences in circulating levels of a biomarker are merely minute, it is difficult to envision how the determination of this marker would help to diagnose a disorder and to improve treatment.

Does it make sense to analyze a biomarker, if GWAS data for the respective gene are negative? The answer is a clear yes: First of all, whereas the causative gene may be close to the statistically associated SNP, it can also be farther removed. If for example, the causal variant is a trans-acting factor that regulates transcription, the regulated gene/s might even be located on a different chromosome [8]. Second, a biomarker can be an element of a pathway involved in the respective disorder; as such, interindividual variation of the specific gene is not a prerequisite for the difference in serum levels between cases and controls. Finally, we need to consider epigenetic phenomena, which in turn could influence serum levels, again without necessarily being based on differences at the level of the DNA sequence.

Because of the clinical association of ADHD with obesity (e.g. [4, 7], Albayrak and coworkers (2013) focused on the 32 independent loci that have been associated with obesity at the genome-wide significant level of $p<5 \times 10^{-8}$ as candidate loci for ADHD. The lead BDNF SNP for obesity revealed a $p$ value well above 0.05 as looked up in a GWAS metaanalysis for ADHD. If current DSM-5 criteria are not to constrain a diagnostic approach based on biology as well as symptomatology [10], we could hypothesize that the detection of differences in serum levels of BDNF in ADHD is rendered possible if body mass index $\left(\mathrm{BMI} ; \mathrm{kg} / \mathrm{m}^{2}\right.$ ) is additionally taken into account. Accordingly, we would need to stratify ADHD patients according to their BMI. Subjects with both ADHD and obesity could represent the ideal case group for such a study. Indeed, BDNF has been implicated in the development of both obesity and ADHD in rodent and 
human studies $[9,11]$. In their study, Scarletti and coworkers described similar BMI values for both cases and controls.

In light of findings suggesting a further role of BDNF in cognition e.g. [2], a further phenotypical dissection could entail ascertaining subjects with hyperactivity, obesity and low cognitive function. We certainly would be leaving the diagnostic boundaries imposed by DSM well behind us. It must, however, be pointed out that several other subdifferentiations based on both biology and symptomatology appear possible thus rendering the identification and confirmation of such a biomarker a daunting task. Whereas the underlying biology clearly warrants such a differentiated approach, ascertainment and phenotyping of a sufficiently large number of patients represents the major limiting factor. Small effect sizes might very well apply even in such patient (and control) samples characterized for both psychiatric and biological phenotypes. Necessary confirmatory studies would require that independent groups have collected comparable data on the same phenotypes of patients with the same disorder. As such, it would appear valuable to discuss those additional phenotypes that appear promising for the elucidation of biomarkers relevant in subgroups of patients with specific psychiatric disorders.

\section{References}

1. Albayrak Ö, Pütter C, Volckmar AL, Cichon S, Hoffmann P, Nöthen MM, Jöckel KH, Schreiber S, Wichmann HE, Faraone SV, Neale BM, Herpertz-Dahlmann B, Lehmkuhl G, Sinzig J, Renner TJ, Romanos M, Warnke A, Lesch KP, Reif A, Schimmelmann BG, Scherag A, Hebebrand J, Hinney A, Psychiatric GWAS Consortium: ADHD Subgroup (2013) Common obesity risk alleles in childhood attention-deficit/hyperactivity disorder. Am J Med Genet B Neuropsychiatr Genet 162B(4):295-305

2. Carlino D, De Vanna M, Tongiorgi E (2013) Is altered BDNF biosynthesis a general feature in patients with cognitive dysfunctions? Neuroscientist 19(4):345-353

3. Corominas-Roso M, Ramos-Quiroga JA, Ribases M, SanchezMora C, Palomar G, Valero S, Bosch R, Casas M (2013) Decreased serum levels of brain-derived neurotrophic factor in adults with attention-deficit hyperactivity disorder. Int J Neuropsychopharmacol 30:1-9

4. Cortese S, Vincenzi B (2012) Obesity and ADHD: clinical and neurobiological implications. Curr Top Behav Neurosci 9:199-218

5. Dalsgaard S (2013) Attention deficit/hyperactivity disorder (ADHD). Eur Child Adolesc Psychiatry (Suppl 1):S43-S48

6. Dudley E, Hässler F, Thome J (2011) Profiling for novel proteomics biomarkers in neurodevelopmental disorders. Expert Rev Proteomics 8(1):127-136
7. Erhart M, Herpertz-Dahlmann B, Wille N, Sawitzky-Rose B, Hölling H, Ravens-Sieberer U (2012) Examining the relationship between attention-deficit/hyperactivity disorder and overweight in children and adolescents. Eur Child Adolesc Psychiatry 21:39-49

8. Gejman PV, Sanders AR, Duan J (2010) The role of genetics in the etiology of schizophrenia. Psychiatr Clin North Am 33(1):35-66

9. Gray J, Yeo GS, Cox JJ, Morton J, Adlam AL, Keogh JM, Yanovski JA, El Gharbawy A, Han JC, Tung YC, Hodges JR, Raymond FL, Órahilly S, Farooqi IS (2006) Hyperphagia, severe obesity, impaired cognitive function, and hyperactivity associated with functional loss of one copy of the brain-derived neurotrophic factor (BDNF) gene. Diabetes 55(12):3366-3371

10. Insel T (2013) Director's Blog: transforming diagnosis. http:// www.nimh.nih.gov/about/director/2013/transforming-diagnosis. shtml

11. Kernie SG, Liebl DJ, Parada LF (2000) BDNF regulates eating behavior and locomotor activity in mice. EMBO $\mathrm{J}$ 19(6):1290-1300

12. Myatchin I, Lemiere J, Danckaerts M, Lagae L (2012) Withinsubject variability during spatial working memory in children with ADHD: an event-related potentials study. Eur Child Adolesc Psychiatry 21:199-210

13. Rout UK, Mungan NK, Dhossche DM (2012) Presence of GAD65 autoantibodies in the serum of children with autism or ADHD. Eur Child Adolesc Psychiatry 21(3):141-147

14. Scassellati C, Bonvicini C, Faraone SV, Gennarelli M (2012) Biomarkers and attention-deficit/hyperactivity disorder: a systematic review and meta-analyses. J Am Acad Child Adolesc Psychiatry 51(10):1003-1019

15. Scassellati C, Zanardini R, Tiberti A, Pezzani M, Valenti V, Effedri P, Filippini E, Conte S, Ottolini A, Gennarelli M, Bocchio-Chiavetto L (2013) Serum brain-derived neurotrophic factor (BDNF) levels in attention deficit-hyperactivity disorder (ADHD). Eur Child Adolesc Psychiatry. doi:10.1007/s00787013-0447-1

16. Schmidt HD, Shelton RC, Duman RS (2011) Functional biomarkers of depression: diagnosis, treatment, and pathophysiology. Neuropsychopharmacology 36:2375-2394

17. Shim SH, Hwangbo Y, Kwon YJ, Jeong HY, Lee BH, Lee HJ, Kim JK (2008) Increased levels of plasma brain-derived neurotrophic factor (BDNF) in children with attention deficit-hyperactivity disorder (ADHD). Prog Neuropsychopharmacol Biol Psychiatry 32:1824-1828

18. Thome J, Ehlis AC, Fallgatter AJ, Krauel K, Lange KW, Riederer $\mathrm{P}$, Romanos M, Taurines R, Tucha O, Uzbekov M, Gerlach M (2012) Biomarkers for attention-deficit/hyperactivity disorder (ADHD). A consensus report of the WFSBP task force on biological markers and the World Federation of ADHD. World J Biol Psychiatry 13(5):379-400

19. Wallis D (2010) The search for biomarkers for attention deficit/ hyperactivity disorder. Drug News Perspect 23(7):438-449. doi:10.1358/dnp.2010.23.7.1472296

20. Zhang L, Chang S, Li Z, Zhang K, Du Y, Ott J, Wang J (2012) ADHD gene: a genetic database for attention deficit hyperactivity disorder. Nucleic Acids Res 40:1003-1009 